\title{
BEHAVIOR AT THE BOUNDARY OF A SOLUTION TO PLATEAU'S PROBLEM ${ }^{1}$
}

\author{
BY JOHN E. BROTHERS \\ Communicated by S. S. Chern, October 12, 1972
}

Knowledge of the existence and structure of tangent cones plays a basic role in the study of the interior structure of area minimizing integral currents $[6, \S 5.4]$ and in the study of the structure of rectifiable varifolds [2]. Moreover, in his study of boundary regularity of area minimizing currents with smooth boundary, William Allard [1] makes extensive use of tangent cones at points on the boundary. It is therefore to be expected that tangent cones will continue to play a central role in the investigation of the behavior at the boundary of area minimizing currents. Here we present results concerning the existence and structure of oriented tangent cones at points on the boundary of an area minimizing integral current. The proofs will appear elsewhere. Terminology and notation will be consistent with that of [6]; see in particular the List of Notations on pp. 670 and 671.

I am indebted to William Allard for many stimulating discussions on this subject, especially with regard to Theorem 1 , which he originally proved under stronger hypotheses.

Our results will be given in terms of the potential theoretic function $V^{\partial S}$ which was first considered by Radon [9] and subsequently was used by $\mathrm{J}$. Král (in the case where $k=n$ and $S$ is obtained by integration over a Borel subset of $\boldsymbol{R}^{n}$ ) in discussing the Neumann boundary value problem and the heat equation for domains with nonsmooth boundary [7], [8]. In [3] we extended the definition of $V^{\partial S}$ to the case where $k$ is arbitrary and $\partial S$ is replaced by a flat current $T$, and investigated the properties of $V^{T}$. Let $B$ be an oriented $(n-k+1)$-dimensional linear subspace of $\boldsymbol{R}^{n}$, $T \in \boldsymbol{F}_{k-1}\left(\boldsymbol{R}^{n}\right), a \in \boldsymbol{R}^{n}$ and $r>0$. We define

$$
V^{T}(a, r)=n \alpha(n) \int_{S O(n)} \boldsymbol{M}\left[T\left[B(a, r) \cap\left(a+g_{\#} B\right)\right] d \Psi g\right.
$$

where $\Psi$ is the Haar measure on $\boldsymbol{S O}(n)$ and $\alpha(n)$ is the volume of the unit ball in $\boldsymbol{R}^{n}$. In case $\boldsymbol{M}(T)<\infty, V^{T}(a, r)$ is proportional to

AMS (MOS) subject classifications (1970). Primary 49F22; Secondary 49F10, 53A10.

Key words and phrases. Integral current, boundary, absolutely area minimizing integral current, oriented cone, oriented tangent cone, Plateau problem.

${ }^{1}$ This work was supported in part by the National Science Foundation. 


$$
\int_{\boldsymbol{B}(a, r)}|x-a|^{-k}|(x-a) \wedge \vec{T}(x)| d\|T\| x .
$$

We set $V^{T}(a, \infty)=V^{T}(a)$. Finally, it follows from the proof of $[3,7.4]$ that if $T$ is an oriented proper $(k-1)$-dimensional submanifold of class 1 whose tangent planes satisfy a Hölder condition in a neighborhood of $a$, then

$$
\int_{0}^{* \infty} \frac{1}{t} V^{T}(a, t) d t<\infty
$$

where " $\int^{*}$ " denotes "upper integral."

We recall that a current $C$ is an oriented cone if and only if

$$
\boldsymbol{\mu}_{\boldsymbol{r} \#} C=C \quad \text { whenever } r>0,
$$

where $\boldsymbol{\mu}_{r}(x)=r x$ for $x \in \boldsymbol{R}^{n}$. Assuming $S \in \mathscr{F}_{k}^{\text {loc }}\left(\boldsymbol{R}^{n}\right)$ (the topological group of locally integral flat currents of dimension $k$ ) and $a \in \boldsymbol{R}^{n}$ we also recall that an oriented cone $C \in \mathscr{F}_{k}{ }_{k}^{\text {loc }}\left(\boldsymbol{R}^{n}\right)$ is an oriented tangent cone of $S$ at $a$ if there exists a sequence of positive numbers $r_{j}$ such that

$$
\lim _{j \rightarrow \infty} r_{j}=\infty \text { and } \lim _{j \rightarrow \infty}\left(\boldsymbol{\mu}_{r_{j}} \circ \tau_{-a}\right)_{\#} S=C \text {. }
$$

(Here $\tau_{-a}(x)=x-a$ for $x \in \boldsymbol{R}^{n}$.)

Whenever $S$ is a $k$-dimensional rectifiable current, one defines the $k$-dimensional density of $S$ at $a$ by

$$
\Theta^{k}(\|S\|, a)=\lim _{r \rightarrow 0} \alpha(k)^{-1} r^{-k}\|S\| \boldsymbol{B}(a, r)
$$

for each $a \in \boldsymbol{R}^{n}$ such that this limit exists.

Let $M$ be a properly embedded submanifold of $\boldsymbol{R}^{n}$ of class 2 . We recall that an integral current $S \in \boldsymbol{I}_{k}\left(\boldsymbol{R}^{n}\right)$ is absolutely area minimizing with respect to $M$ if spt $S \subset M$ and $M(S) \leqq M\left(S^{\prime}\right)$ whenever $S^{\prime} \in I_{k}\left(R^{n}\right)$ with spt $S^{\prime} \subset M$ and $\partial S=\partial S^{\prime}$. Further, $S \in \boldsymbol{I}_{k}^{\text {loc }}\left(\boldsymbol{R}^{n}\right)$ is absolutely area minimizing with respect to $M$ if $S[K$ is absolutely area minimizing with respect to $M$ for each compact subset $K$ of $\boldsymbol{R}^{n}$.

THEOREM 1. Assume $S \in \boldsymbol{I}_{\boldsymbol{k}}\left(\boldsymbol{R}^{n}\right)$ is absolutely area minimizing with respect to $M$. Then, at $\|\partial S\|$ almost all $a \in \operatorname{spt} \partial S$ such that $V^{\partial S}(a)<\infty$, there exists an oriented tangent cone of $S$. Moreover, if $C$ is an oriented tangent cone of $S$ at $a$, then

(i) $C \in \boldsymbol{I}_{k}^{\text {loc }}\left(\boldsymbol{R}^{n}\right)$ and $C$ absolutely minimizes area with respect to $\boldsymbol{R}^{n}$;

(ii) $\partial C=\theta P$, where $\theta$ is a positive integer and $P$ is an oriented $(k-1)$ dimensional linear subspace of $\boldsymbol{R}^{n}$;

(iii) $\Theta^{k}(\|S\|, a)=\Theta^{k}(\|C\|, 0) \geqq \Theta^{k}(\|C\|, x)$ for $x \in P$. 
In demonstrating the existence of a tangent cone, we first show that $\Theta^{k}(\|S\|, a)$ is a real number using an argument involving computation of the first variation of $S$ under the radial deformation

$$
h(t, z)=\left[1+t|z-a|^{-k}\right](z-a) \text { for }(t, z) \in \boldsymbol{R} \times \boldsymbol{R}^{n} \sim\{a\} .
$$

We then proceed in a manner similar to that used in the proof of $[6,5.4 .3]$ to obtain the analogous result for the interior problem.

In case there exists a proper $(k-1)$-dimensional submanifold $B$ of class 1 such that the tangent spaces of $B$ satisfy a Hölder condition and

$$
\|\partial S\|\left[U=\theta \mathscr{H}^{k-1}\lfloor B\right.
$$

for some open set $U$ and some positive integer $\theta$, then the conclusions of Theorem 1 hold for each $a \in B$.

THeOREM 2. Assume $S \in \boldsymbol{I}_{k}\left(\boldsymbol{R}^{n}\right)$ is absolutely area minimizing with respect to $M$. Then, for $\|\partial S\|$ almost all $a \in \operatorname{spt} \partial S$ such that

$$
\int_{0}^{* \infty} \frac{1}{t} V^{\partial S}(a, t) d t<\infty
$$

the conclusions of Theorem 1 are true with equality holding in (iii) whenever $x \in P$.

The proof is based on results of [3], [4], [5] and on application of the variational formula $[2,5.1(1)]$, as is also the proof of the following:

THEOREM 3. Let $P$ be an oriented $(k-1)$-dimensional linear subspace of $\boldsymbol{R}^{n}, \theta$ be a positive integer, and $C \in \boldsymbol{I}_{k}^{\text {loc }}\left(\boldsymbol{R}^{n}\right)$ be an absolutely area minimizing oriented cone such that $\partial C=\theta P$ and $\Theta^{k}(\|C\|, x)$ is constant for $x \in P$. Then there exist distinct oriented $k$-dimensional closed half-planes $Q_{1}, \ldots, Q_{m}$ and integers $\alpha_{1}, \ldots, \alpha_{m}$ such that

$$
\begin{gathered}
\partial Q_{i}=P \quad \text { for } i=1, \ldots, m, \\
C=\alpha_{1} Q_{1}+\cdots+\alpha_{m} Q_{m} \text { and } \theta=\alpha_{1}+\cdots+\alpha_{m} .
\end{gathered}
$$

Furthermore, if $\alpha_{i}>0, i=1, \ldots, m$, then

$$
\Theta^{k}(\|C\|, 0)=\frac{1}{2} \theta ;
$$

otherwise, $m=2$ and spt $C=Q_{1} \cup Q_{2}$ is a linear subspace of $\boldsymbol{R}^{n}$ with

$$
\Theta^{k}(\|C\|, 0)=\frac{1}{2}\left|\alpha_{1}-\alpha_{2}\right| \text {. }
$$

Theorems 2 and 3 completely characterize the structure of tangent cones to $S$ at $\|\partial S\|$ almost all $a \in \boldsymbol{R}^{n}$ such that the integral (*) is finite. We 
conclude by presenting some partial results concerning the structure of oriented cones for which the hypotheses of Theorem 3 do not hold. Theorem 1 can be extended to obtain the existence of an area minimizing tangent cone of $S$ at a "corner" of $\partial S$ where $\partial S$ has a tangent cone whose support is not a $(k-1)$-plane. In this situation Theorem 4 is relevant. In case $C \in \boldsymbol{I}_{\boldsymbol{k}}^{\text {loc }}\left(\boldsymbol{R}^{n}\right)$ is an absolutely area minimizing oriented cone such that $\partial C$ is a $(k-1)$-plane, one can show with the help of Theorem 1 that

$$
\Theta^{k}(\|C\|, x) \leqq \Theta^{k}(\|C\|, 0) \text { for each } x \in \operatorname{spt} \partial C .
$$

We do not know whether there exists $C$ for which inequality holds at some $x \in \operatorname{spt} \partial C$.

Let $C \in \boldsymbol{I}_{k}^{\text {loc }}\left(\boldsymbol{R}^{n}\right)$ be an oriented cone such that $\partial C=\theta P$ where $\theta$ is a positive integer and $P$ is an oriented $(k-1)$-dimensional linear subspace of $\boldsymbol{R}^{n}$. Then $[\mathbf{4}, 3.6]$ implies that

$$
\theta \leqq 2 \Theta^{k}(\|C\|, 0),
$$

with equality holding if and only if $C \cap S^{n-1}$ absolutely minimizes area with respect to $S^{n-1}$, in which case $C$ absolutely minimizes area with respect to $\boldsymbol{R}^{n}$,

$$
\theta=2 \Theta^{k}(\|C\|, b) \text { for } b \in P,
$$

and Theorem 3 gives the structure of $P$. More generally, we have the following:

THEOREM 4. Suppose $C \in \boldsymbol{I}_{k}^{\text {loc }}\left(\boldsymbol{R}^{n}\right)$ is an oriented cone such that $C \cap \boldsymbol{S}^{n-1}$ absolutely minimizes area with respect to $S^{n-1}$ (hence $C$ absolutely minimizes area with respect to $\boldsymbol{R}^{n}$ ) and there exists a closed, oriented $k$-dimensional half-plane $Q_{0}$ with spt $\partial C \subset Q_{0}$. Denote $P=\partial Q_{0}$. Then there exist oriented $k$-dimensional half-planes $Q_{1}, \ldots, Q_{m}$ and nonnegative integers $\alpha_{1}, \ldots, \alpha_{m}$ such that

(i) $Q_{j} \neq Q_{0}$ and $\partial Q_{j}=P$ for $j=1, \ldots, m$, and

$$
C=\beta\left(\alpha_{1} Q_{1}+\cdots+\alpha_{m} Q_{m}\right)+C\left\lfloor Q_{0}, \quad \beta= \pm 1\right.
$$

(ii) If spt $\partial C \subset P$, then $C\left\lfloor Q_{0}=\beta \alpha_{0} Q_{0}\right.$, where $\alpha_{0}$ is a nonnegative integer. Moreover, $\partial C=\theta P, \beta=\operatorname{sign} \theta, \theta=\alpha_{0}+\alpha_{1}+\cdots+\alpha_{m}$ and $\Theta^{k}(\|C\|, 0)=\frac{1}{2} \theta$.

(iii) If $P \not \subset$ spt $\partial C$, then spt $C \subset Q_{0}$.

The final result which we will present was communicated to us by William Allard.

THEOREM 5. Let $C \in \boldsymbol{I}_{k}^{\text {loc }}\left(\boldsymbol{R}^{n}\right)$ be an oriented cone such that $\partial C=\theta P$, where $P$ is an oriented $(k-1)$-dimensional linear subspace of $\boldsymbol{R}^{n}$ and $\theta$ is 
a positive integer. Suppose that

$$
\left.\frac{d}{d t} \boldsymbol{M}\left(h_{t \#} C\right)\right|_{t=0}=0
$$

whenever $h$ is a smooth isotopy of $\boldsymbol{R}^{n}$ with compact support such that $h_{t \#} P=P$ for all $t \in \boldsymbol{R}$. (In particular this holds if $C$ absolutely minimizes area.) If spt $C \sim P$ lies in an open half-space of $\boldsymbol{R}^{n}$, then there exist oriented $k$-dimensional half-planes $Q_{1}, \ldots, Q_{m}$ and positive integers $\alpha_{1}, \ldots, \alpha_{m}$ such that $\partial Q_{i}=P$ for $i=1, \ldots, m$,

$$
C=\alpha_{1} Q_{1}+\cdots+\alpha_{m} Q_{m} \text { and } \theta=\alpha_{1}+\cdots+\alpha_{m} .
$$

ADDED IN PROOF. We have recently shown that the condition (*) in the hypothesis of Theorem 2 can be replaced by $V^{\partial s}(a)<\infty$.

\section{REFERENCES}

1. W. K. Allard, On boundary regularity for Plateau's problem, Bull. Amer. Math. Soc. 75 (1969), 522-523. MR 39 \# 3140.

2. - On the first variation of a varifold, Ann. of Math (2) 95 (1972), 417-491.

3. J. E. Brothers, A characterization of integral currents, Trans. Amer. Math. Soc. 150 (1970), 301-325. MR 42 \# 1034.

4. - Stokes' theorem, Amer. J. Math. 92 (1970), 657-670. MR 43 \# 486.

5. - Stokes' theorem. II, Amer. J. Math. 93 (1971), 479-484.

6. H. Federer, Geometric measure theory, Die Grundlehren der math. Wissenschaften, Band 153, Springer-Verlag, New York, 1969. MR 41 \# 1976.

7. J. Král, The Fredholm method in potential theory, Trans. Amer. Math. Soc. 125 (1966), 511-547, MR 35 \# 4006.

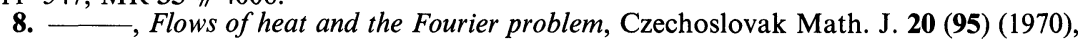
556-598. MR 42 \#6437.

9. J. Radon, Über die Randwertaufgaben bein logarithmischen Potential, S.-B. Akad. Wiss. Wien. 128 (1919), 1123-1167.

DePartment of Mathematics, Indiana University, BloOmington, Indiana 47401 